\title{
Titanium surface modified by nitrogen ion implantation
}

\author{
Jan Drahokoupil' ${ }^{1}$ Petr Vlčák ${ }^{2}$, Miroslav Lebeda ${ }^{1,2}$ \\ ${ }^{I}$ Institute of Physics, AS CR, Na Slovance 2, 18221 Prague, Czech Republic; \\ ${ }^{2}$ Faculty of Mechanical Engineering, Czech Technical University in Prague, Technicka 4, 16607 Prague, Czech Republic; \\ draho@fzu.cz
}

Titanium alloys are material that is often used in aerospace industry and in biomedical engineering for their excellent properties as low density, high tensile strength and corrosion resistance in some common environments. On the other hands the disadvantages of these materials are in general poor performance in sliding, hardness and wear. Enriching the surface area of pure titanium by nitrogen lead to improve hardness, corrosion resistance and abrasion resistance. Moreover, the required low modulus of elasticity of bulk material is maintained which is susscesfully used for example in joint replacements and dental crowns.

In our contribution we would like to present the study of surface area of alfa-titanium after implantation of nitrogen ions. The X-ray diffraction and x-ray reflectivity were used for characterization of these surfaces. The special cheap home-made improvement of incident beam that enable to reach lower angles during reflectivity measurements will be presented.

Keywords: titanium, $x$-ray reflectivity, surface, ion implantation 